\title{
A Thin Slice Type High Stabilization Optical Current Sensor
}

\author{
Shixin $\mathrm{Jin}^{1, \mathrm{a}}$, Tongwei $\mathrm{Yu}^{1, \mathrm{~b}}$, Ziliang $\mathrm{Li}^{1, \mathrm{c}}$ and Xun $\mathrm{MaO}^{2, \mathrm{~d}}$ \\ ${ }^{1}$ Electric Power Research Institute, State Grid Liaoning Electric Power Co., Ltd., Shenyang 110000, \\ China; \\ ${ }^{2}$ School of Electrical and Electronic Engineering, North China Electric Power University, Baoding \\ 071003, China. \\ ajsxmumu@126.com, byu_twei@163.com, 'lizl_ldk@126.com, datston_mx@126.com
}

\begin{abstract}
Keywords: optical current transducer, Faraday Effect, magneto-optical material, sensitivity, linear birefringence.
\end{abstract}

\begin{abstract}
Optical current sensor has a wide range of applications. The main factors that affect the practical process of optical current transducer (OCT) are the effects of birefringence, temperature and long-term stability. Optical current transducer (OCT) with thin slice type sensing head is designed in this paper. Due to the small size of the sensing element of sensing head, the influence of the transient birefringence caused by temperature gradient is negligible. The long-term operation stability of the system is improved by using the non bonding package; the measurement sensitivity of the system is improved by using a new type of magneto-optical material BiGd: YIG. The experimental system used LD as light source, PIN as photoelectric detector, and PC to acquire output signal and data process. The experimental results show that the output signal is linear with the magnetic field in the range of 1909.19Oe, and the feasibility of this design is verified.
\end{abstract}

\section{Introduction}

Optical current transducer (OCT) has many potential benefits over conventional current transducer, such as strong ability to anti electromagnetic interference, wide dynamic range, relatively simple of the insulation system, easy to interface with computer [1]. Although OCT has a wide range of applications as the ideal alternative to traditional current transducer [2], the technology is still imperfect. The influence of birefringence, temperature and long-term stability are the main problems in the development of optical current transducer. The birefringence of the sensing system is an important reason to affect the sensitivity and accuracy of the system. However, the effect of the transient birefringence caused by temperature has complex random properties which are difficult to find an effective method to solve it. This problem is one of the key technical difficulties in the practical application of optical current transducer.

In order to reduce the influence of transient birefringence, the optical current sensor designed in this paper requires a short optical path of optical sensor, that is to say the size of the magneto-optical material is small. However, the short optical path can reduce the sensitivity of the optical sensing head, so as to reduce the output signal to noise ratio of the sensor system and to influence the measurement accuracy. In order to coordinate the relationship between the suppression of transient birefringence and the improvement of the sensitivity of the system, magneto-optical material with good sensitivity and high Verdet constant is selected.

\section{Sensing Principle and Stability of Optical Current Transducer}

OCT based on Faraday magneto-optical effect measuring the current indirectly by measuring the magnetic field caused by measured current. The external magnetic field added on the magneto-optical material will cause the rotation of the polarization plane of the polarized light through the magneto-optical material. The relationship between the deflection angle $\theta$ and the external magnetic field is: 


$$
\theta=\mu \cdot V \cdot \int_{\mathrm{L}} \vec{H} \cdot d \vec{l}
$$

Where $\mu$ is the magnetic permeability of Faraday magneto-optical material, $\mathrm{V}$ is the Verdet constant of magneto-optical material which is related to the characteristics of the media, the wavelength of light source and external temperature, $\mathrm{H}$ is the magnetic field intensity acting on the magneto-optical material and $\mathrm{L}$ is the optical path length of the polarized light through magneto-optical material.

Since the deflection angle of polarized light cannot be measured directly, we use the analyzer to detect the light intensity signal. Select reference coordinate system to make the transmission axis of the analyzer parallel to the $\mathrm{X}$ axis. The detected sensitivity is highest when the angle between the analyzer and the polarizer is $\pi / 4$. Then the relationship between the output light intensity $p_{o}$ and the input light intensity $P_{i}$ is

$$
p_{o}=\frac{1}{2} P_{i}(1+\sin 2 \theta)
$$

$\mathrm{AC}$ and DC components are detected by the filter circuit. To eliminate the influence of the fluctuation of input light intensity, the modulated quantity is taken as

$$
m=P_{A C} / P_{D C}=\sin 2 \theta
$$

The modulated quantity is small. $m \approx 2 \theta$.

Multiple optical components are needed in the bulk OCT in order to form a closed optical path. Optical adhesive bonding is required between each optical element. The light is transmitted in the sensing head through multiple reflections which is transmitted by the optical bonding surface. The influences of Optical adhesive bond are mainly the following: The transmission efficiency of optical adhesive is a function of temperature and time, the effect of temperature on the optical adhesive bonding surface will lead to the ideal optical path deviation which affects the coupling efficiency of the output lens to the optical fiber. The problem of operation stability of block OCT will appear after a period of time due to the use of optical adhesive.

Long optical path and multi loop are the main reasons for the problem of long-term operation stability of OCT, but also become one of the main reasons to hinder the practicality of OCT [3]. Therefore, the key to solve the problem of the long-term operation stability of Faraday magneto-optical effect OCT is to simplify the optical path and to use the optical adhesive bonding as little as possible, which can be realized by using the sensing head of straight-type structure. The straight-type sensing head without reflection prism is composed of three parts, a polarizer, a magneto-optic glass and a polarizing optical splitter. The three optical axes are in a straight line and not bonded to each other.

The effect of temperature on the current transducer is mainly reflected in the accuracy of the sensing head. The effect can be divided into the following two influences of temperature source according to the actual situation: one is ambient temperature that spatially uniformly distributed and changing slowly; another is the temperature that spatially inhomogeneous distributed and changing complex. In the uniform temperature field, light intensity that transmitted in the sensing head changes, and then the optical power changes. This effect can be solved by using appropriate data processing method. In the non-uniform temperature field, the uneven thermal expansion of the magneto-optical material and optical device in the sensing head is caused by the uneven temperature of sensing head, then causing the internal stress and additional temperature stress birefringence, directly affecting the deflection angle of the polarized light, resulting in the measurement errors which are difficult to eliminate. Uneven temperature not only produces birefringence, but also affects Verdet constant of the medium, refractive index, etc.

\section{The design of thin slice type high sensitivity sensor system}

The Selection of the Magneto-Optical material. Reducing the size of sensing head and simplifying optical path can reduce the effect of transient birefringence greatly[4]. In addition, the 
temperature gradient in the sensing material can be reduced by reducing the size of the magneto-optical material, and the thermal stress generated by the temperature changes will also be reduced.

In order to reduce the influence of transient birefringence, the optical current sensor designed in this paper is required for the short optical path of the optical sensor part, that is, the small size of the magneto-optical material. However, shorting the optical path can reduce the sensitivity of the optical sensing head, so as to reduce the output signal to noise ratio of the sensor system, and to influence the measurement accuracy. Magneto-optical material of high Verdet constant and high sensitivity should be choose in order to coordinate the relationship between the suppression of transient birefringence and the improvement of the sensitivity of the system.

YIG series of rare earth iron garnet magneto-optic crystal with large Faraday Effect, small light absorption, good transparency, good physical and chemical properties in the near infrared band of more than $1 \mathrm{~m}$, is very suitable for optical current sensor. A new type of high sensitivity magneto-optical material BiGd: YIG is selected in this paper. YIG with large Verdet constant and good properties of crystal is a good Faraday magneto-optical material, in which the incorporation of GD can reduce the temperature dependence; the incorporation of $\mathrm{Bi}$ can improve the sensitivity of Faraday magneto-optical effect [5].

Configuration of Thin Slice Type Optical Current Sensor. Faraday magneto-optical material of thin slice type optical current sensor is only $0.1 \mathrm{~mm}$ in size. The straight-type sensing head without reflection prism is composed of three parts, a polarizer, a magneto-optic glass and a polarizing optical splitter. The three optical axes are in a straight line and not bonded to each other.

The specific optical path structure is: the light emitted by the light source is turned into parallel light by a self focusing lens, and the parallel light is changed into a linear polarized light by a polarizer, then the linear polarized light is incident to the sensitive element. The light in the sensitive element is deflected under the action of the measured current; the emitted modulated light output to the detector, the transmission light of the analyzer output to the coupled self focusing lens, then the light is transmitted to the photoelectric detector, polarizer and analyzer by the optical fiber. The angle between polarizer direction and analyzer direction is $\pi / 4$. At this point, the output intensity of the Faraday rotation angle $\theta$ is the most sensitive, linear degree is the best, the dynamic range is the largest.

Sensitive element: BiGd:YIG, size: $5 * 5 * 0.1 \mathrm{~mm}$. The package size of the entire sensing head is $\Phi 5.5 \mathrm{~mm} \times 34 \mathrm{~mm}$. Figure 1 is the photo of sensor package.

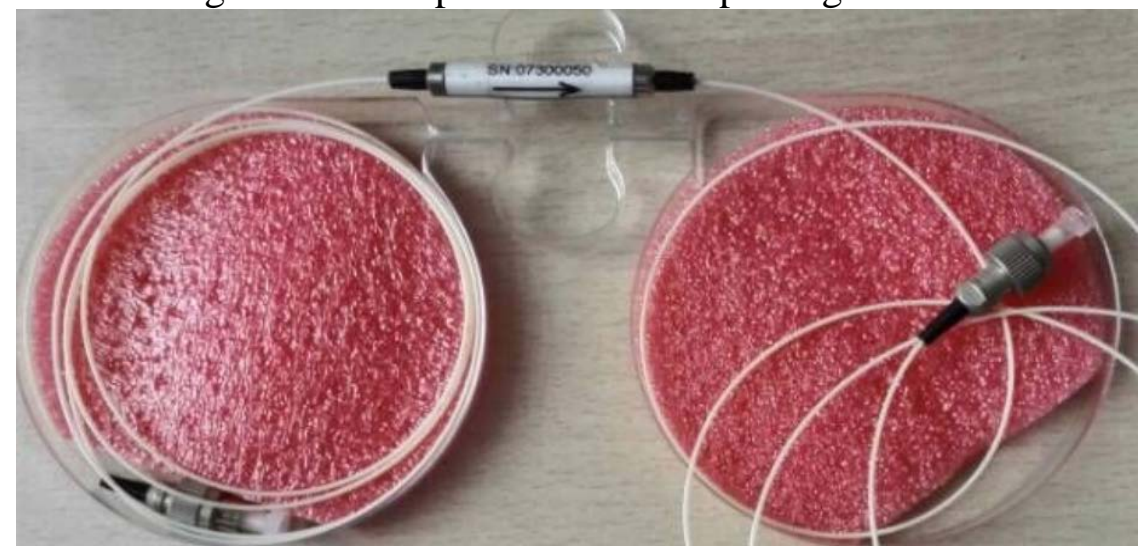

Fig. 1 The photo of sensor package

Experimental Setup. The structure of the experimental system is shown in Figure 2, which includes the light source part, the Faraday rotation part, the photoelectric conversion part, the signal processing part, and the part to produce the measured current. The variable transformer generates current signal, and then the coil and the standard resistor are connected in series to form a current loop. Optical sensing head is placed in the center of the coil. The laser from LD is transmitted to the sensing head by the optical fiber, after the coupling of the self focusing lens, changing into line polarized light through polarizing prism, modulated by magnetic field produced by the measured current. Then the 
light polarization state is detected by a polarizing prism and transformed into the corresponding light intensity change signal. Finally, the light is transmitted to the photoelectric detector by the optical fiber. The photoelectric detector converts the light intensity signal into electrical signal, which is sent to the acquisition card after a simple pre-amplification and so on.

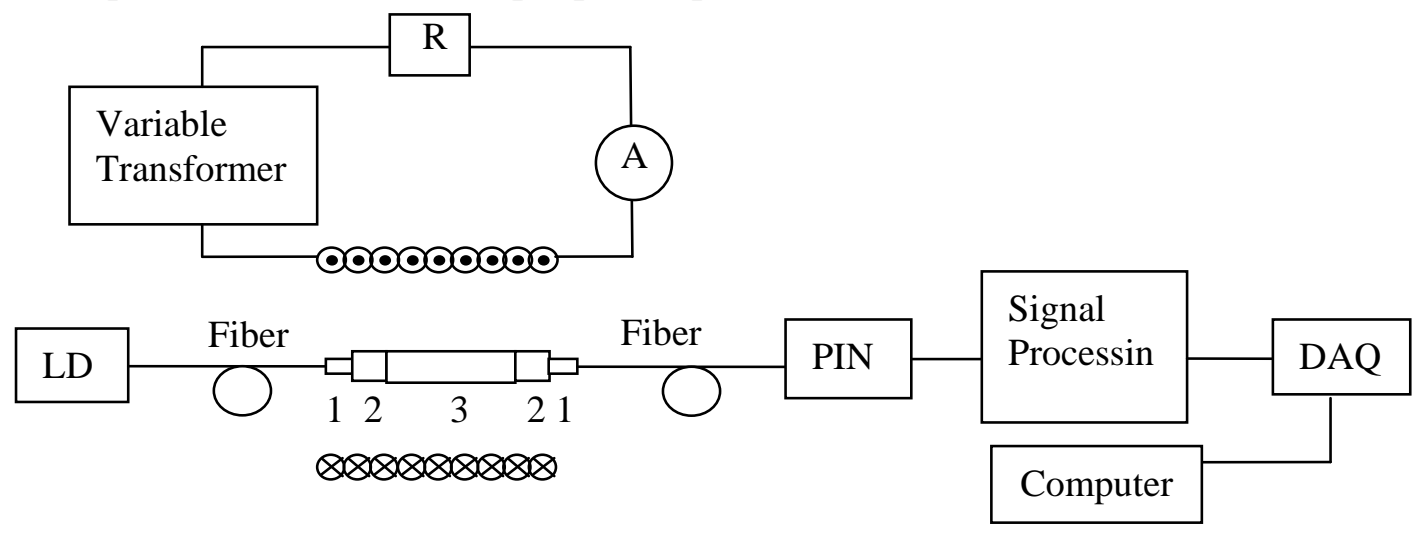

1-Grin Lens 2-Polarizing prism 3-magneto-optic glass

Fig. 2 Experimental system setup

The sensing element is BiGd:YIG. The absorption of this series of crystal in more than $1.0 \mu \mathrm{m}$ of the band is small, about $2 \sim 5 \mathrm{~cm}^{-1}$. So choose the light source of $1.55 \mu \mathrm{m}$ wavelength. PIN30713 type photoelectric diode is produced by the company of Limited Liability Company. This detector is FC series of detectors, coupling by PIN InGaAs photodiode of the $\varphi 300 \mu \mathrm{m}$ photosensitive surface and FC connector through the lens, which can be connected with an optical fiber connector. The circuit has a pin which is easy to connect with electronic components.

The signal processing circuit includes three parts: the photoelectric conversion part, the preamplifier part, the band-pass filter. Operational amplifier uses OP07 of high speed, low noise and high precision. The optical signal carrying the measured current information is projected onto the photoelectric detector and converted into electrical signal. After a simple preprocessing such as amplification and filtering, the signal is sampled by high performance A/D, then the measured current is obtained through data fitting analysis of the digital signal in the PC machine.

\section{Experimental Results}

Determination of the Verdet Constant. The Verdet constant for all magneto-optical materials were determined in this paper. According to the formula $\theta=V H L$, as long as the magnetic field intensity $\mathrm{H}$ and the Faraday rotation angle in this magnetic field intensity is measured, the Verdet constant can be roughly calculated by the above formula.

Experiment of Sensing Head Optical Power Attenuation. The optical power attenuation of the sensing head is measured when the wavelength of light source is $1.31 \mu \mathrm{m}$ and $1.55 \mu \mathrm{m}$ respectively. Test results are shown in table 1:

Table 1 Sensing head optical power attenuation

\begin{tabular}{cccc}
\hline Wavelength & $\begin{array}{c}\text { Optical } \\
\text { power of } \\
\text { light source }\end{array}$ & $\begin{array}{c}\text { Output power } \\
\text { of Sensor } \\
\text { system }\end{array}$ & $\begin{array}{c}\text { attenuation of } \\
\text { optical power }\end{array}$ \\
\hline $1.31 \mu \mathrm{m}$ & $-14 \mathrm{dBm}$ & $-44 \mathrm{dBm}$ & $30 \mathrm{dBm}$ \\
$1.55 \mu \mathrm{m}$ & $-5 \mathrm{dBm}$ & $-7 \mathrm{dBm}$ & $2 \mathrm{dBm}$ \\
\hline
\end{tabular}


After calculation, the optical power attenuation of the $1.55 \mu \mathrm{m}$ wavelength light source is $63.1 \%$. The angle between polarizer and analyzer is $\pi / 4$ in the sensing head which has introduced $50 \%$ loss, so the insertion loss is $13.1 \%$.

Linearity Experiment. The sensing performance of the sensing system is measured given the power frequency alternating current of $50 \mathrm{~Hz}$. The current in the coil is $1 \mathrm{~A} \sim 5 \mathrm{~A}$, and the turns of coil is 1300. The output optical signal of the optical sensor system is converted to electrical signal by PIN component. Signals are acquired and processed by computer after amplification and filtering. The relationship between the output voltage and the coil current is shown in Figure 3:

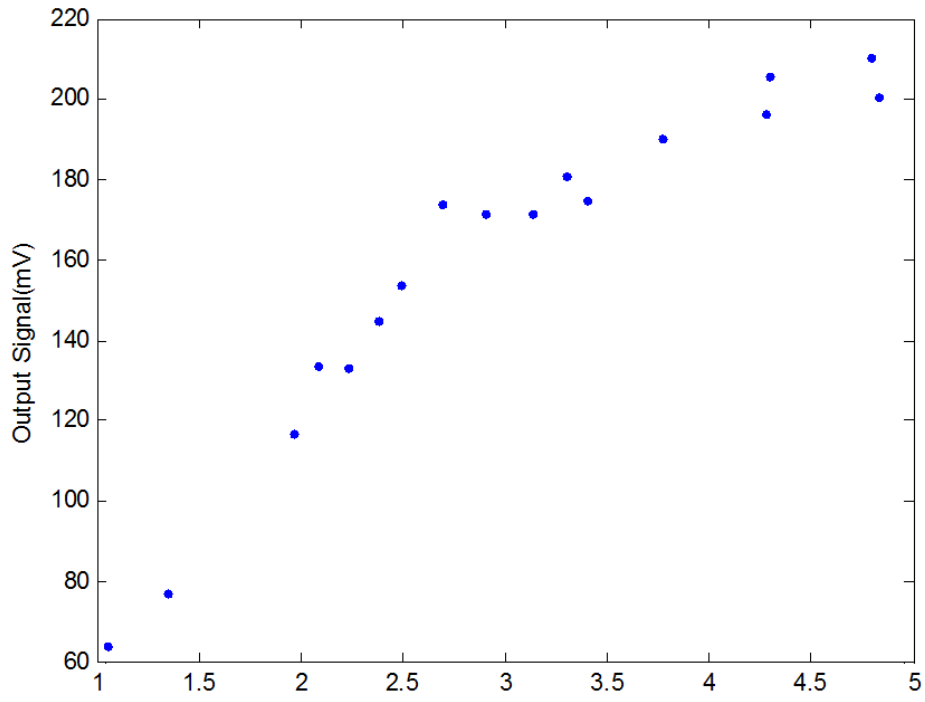

Fig. 3 the relationship between the output signal of the sensor system and the coil current

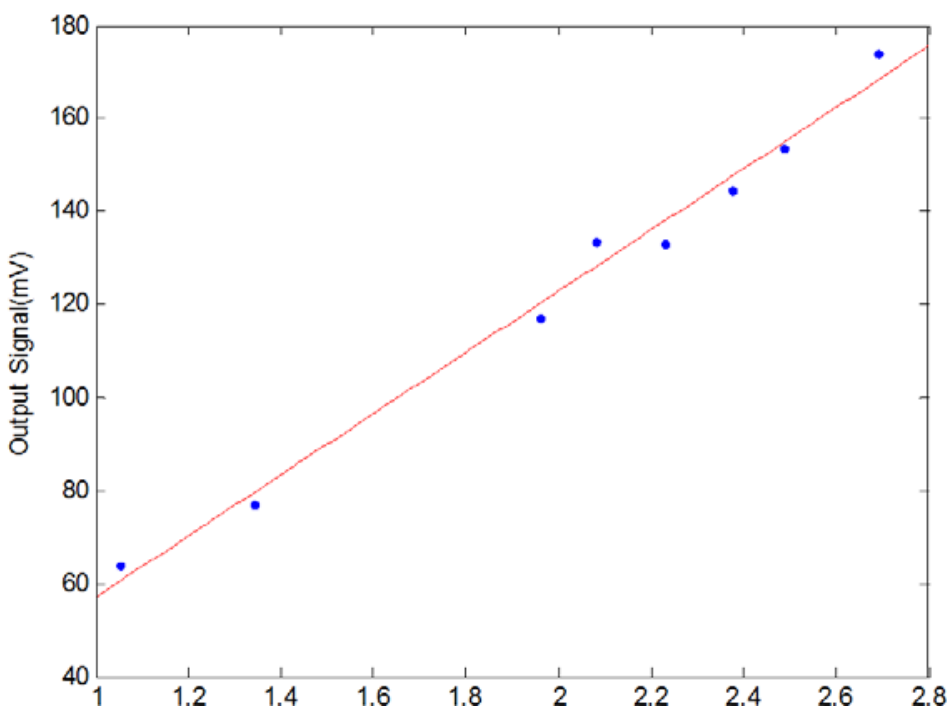

Fig. 4 the relationship between the output signal of the sensor system and the coil current

As can be seen from Figure 3, the current value of 2.689A corresponding to the point of inflection. Before the inflection point, the points are linear, and the later points tends to be flat, which shows that the sensor system reaches magnetic saturation after 2.689A. The magnetic field intensity at the center of the coil is about 1909.190e at 2.689A after measurement. A linear analysis of the current below the point of 2.689A is shown in Figure 4. From Figure 4, the output signal and the coil current are linear basically, and the maximum linear error is 5.7269\%.

System Error Analysis. There are some errors in the test results of this experimental system. The errors are mainly caused by the experimental method and the experimental equipment. The noise of the source is mainly caused by the light source driving circuit. In addition, the intensity noise of the light source will also give the results of measurement interference. The intensity noise of the source is mainly caused by the power fluctuation caused by the environmental impact of the output power of the light source. 
The noise in the circuit mainly has three major components:

(1) Photodiode noise. This part Due to the use of the anti bias circuit, the noise of the photoelectric diode is the sum of the thermal noise (Thermal noise is generated by the reverse junction resistance associated with the dark current and the photocurrent) and the light current. Mean square noise current of this part is $\overline{I_{\mathrm{ns}}^{2}}=2 \mathrm{e}\left(I_{p}+I_{d}\right) B$. Where e is electron charge; $I_{p}$ and $I_{d}$ are respectively the light current and dark current; $\mathrm{B}$ is the sensor bandwidth.

(2) Thermal noise generated by load resistance. The thermal noise current value of this part is: $\overline{I_{\mathrm{nt}}^{2}}=\frac{4 \mathrm{KTB}}{R_{L}}$

(3) Amplifier noise. This noise is determined by the method of using the amplifier and is a function of frequency. In addition, the heat of the coil is another important reason for the error. When the coil is energized, the temperature will rise in a short time, and the temperature of the sensing head in the middle of the coil will increase. In the process of the experiment, the sensing head can be obviously felt the high temperature of the sensor. The Verdet constant of magneto-optical material will change with the change of temperature. The refractive index of other optical devices such as optical fiber is also affected by temperature, which makes the experimental data diverge and brings errors to the experimental results.

\section{Summary}

In this paper, BiGd: YIG is used as the sensitive element of the sensing head. The Verdet constant of magneto-optical material, optical attenuation power of the sensing head and the linearity of the system are tested respectively. Although the results of the experiment cannot reach the ideal value, but the magnetic field intensity is in the range of 1909.190e, the output signal has a linear relationship with the magnetic field, which shows that the scheme of thin slice type sensing head is feasible.

\section{Acknowledgments}

The paper leading to these results has received funding from the science and technology project of State Grid Liaoning Electric Power Co., Ltd. under grant number 2015YF-07.

\section{References}

[1] T.W.Cease, Paul Johnston, R.A. Lupton, A Magneto-Optic Current Transformer, J. Sci. IEEE Trans.on Power Delivery. 5 (1990) 548-555.

[2] K. Barczak, Optical fibre current sensor for electrical power engineering. Technical Sciences. 59(2012)409-414.

[3] Guoqing Zhang, Theory and practical research of optical current transducer, Harbin Institute of Technology. 2005.

[4] Peng Zu, Chi Chiu Chan, Wen Siang Lew et al, Magneto-optical fiber sensor based on magnetic fluid. Optics Letters. 37(2012) 398-400.

[5] Weizhong Zhao, Study on the performance of YbBi: YIG magneto optic current sensor. Journal of infrared and millimeter wave. 20(2001)244-248. 